\title{
AVALIAÇÃO DA FLEXIBILIDADE EM GESTANTES DO ÚLTIMO TRIMESTRE GESTACIONAL
}

Jessica Jessica Ribeiro, Letícia Endsfeldz Teixeira, Nathalia Ulices Savian, Elisa Bizetti Pelai, Alessandra Madia Montovani, Valeria Andrea Alegria Larrua, Andressa Carvalho Viscone, Mariana Romanholi Palma, Edna Maria do Carmo, Cristina Elena Prado Teles Fregonesi

Curso de Fisioterapia da Universidade Estadual Paulista Júlio de Mesquita Filho - Faculdade de Ciências e Tecnologia FCT/UNESP.E-mail: jjr.mendes@hotmail.com

\section{RESUMO}

Durante o período gestacional ocorrem adaptações corporais que alteram a estática, o equilíbrio e a flexibilidade da gestante podendo levar a sobrecarga física, limitações das atividades de vida diária e diminuição da qualidade de vida. O presente estudo tem como objetivo mensurar o grau de flexibilidade de gestantes do sexto ao nono mês, conforme as modificações do período gestacional. $\mathrm{O}$ grupo gestante e o grupo controle passaram por uma avaliação inicial para coleta de dados pessoais e antropométricos e, posteriormente foram avaliados quanto à flexibilidade, por meio dos testes de Banco de Wells e Índice de Schober. Os resultados demonstraram que não houve diferenças entre os grupos tanto em relação à atividade física quanto ao Indice de Schober, no entanto, houve diferenças extremamente significativas no teste de Banco de Wells. A flexibilidade é fundamental para um bom desempenho físico e pode influenciar a qualidade de vida de gestantes.

Palavras-chave: Gestantes, Avaliação, Flexibilidade, Amplitude de Movimento Articular, Fisioterapia.

\section{INTRODUÇÃO}

O período gestacional compreende diversas mudanças corporais ao longo de aproximadamente 36 semanas, segundo necessidades funcionais e metabólicas (GAZANEO, OLIVEIRA, 1998). Essas alterações, inicialmente, decorrem de ações hormonais advindas do desenvolvimento do corpo lúteo e da placenta e, a partir do segundo trimestre, também do crescimento uterino (REIS, 1993; MANN et al., 2008).

As alterações hormonais provocam maior flexibilidade e extensibilidade das articulações, além de promover a retenção hídrica (FABRIN et al., 2010). O aumento da mobilidade dos ligamentos das articulações pélvicas, provocado por elevação dos níveis séricos do hormônio relaxina, pode estar relacionado à dor lombar e pélvica e disfunções sacro-ilíacas, resultando em articulações mais instáveis que podem predispor a lesões (GAZANEO, OLIVEIRA, 1998).

A preparação do corpo para a gestação envolve ajustes dos mais variados sistemas. Os ajustes no sistema postural provenientes do crescimento do útero resultam em protusão abdominal, deslocamento superior do diafragma, mudanças compensatórias na biomecânica da coluna vertebral e rotação pélvica (MANN et al., 2010). 
O crescimento das mamas associado ao alargamento do útero contribui para o deslocamento do centro de gravidade para frente, o que pode acentuar a lordose lombar. Para compensar, a coluna cervical, o quadril e os joelhos adotam a extensão, havendo uma anteriorização da cabeça, seguindo o alinhamento dos ombros em protusão (BIM et al., 2002; MANN et al., 2008).

Essas adaptações acarretam na perturbação das curvas fisiológicas da coluna, anteriorização da pelve e rotação externa dos membros inferiores, levando a gestante a caminhar com uma base de sustentação mais larga (MOREIRA et al., 2011).

Essas alterações, em conjunto com desconfortos músculo-esqueléticos, podem resultar em limitações das atividades diárias e têm implicações consideráveis para as gestantes e para a sociedade em termos da qualidade de vida e produtividade (NASCIMENTO et al., 2011).

Assim, a manutenção da flexibilidade é de suma importância na gestação para equilibrar a musculatura estabilizadora da região dorsal e lombar, abdominal e do assoalho pélvico, uma vez que os músculos dessas regiões estão contraídos continuamente devido à adoção da postura gravídica (LEITÃo et al., 2000).

Baseado no exposto acima parece viável a percepção de que as alterações fisiológicas e biomecânicas possam resultar em modificações na flexibilidade de gestantes. Logo, é fundamental a aplicação de testes para a mensuração da flexibilidade, que abrangem essa variável de forma tridimensional e exploram os diferentes eixos de movimento.

Em virtude de uma literatura escassa sobre esta temática e da relevância do tema para contribuição do desenvolvimento de atividades preventivas próprias para gestantes, à verificação do grau de flexibilidade dessa população se faz necessária, visto que a atividade de vida diária na gestação é limitada. Diante desse contexto, o objetivo do estudo foi mensurar o grau de flexibilidade de gestantes durante o último trimestre de gestação.

\section{MÉTODOS}

Foram selecionadas 30 participantes do gênero feminino com faixa etária de 17 a 35 anos, sendo divididas em dois grupos: GG formado por 15 gestantes do sétimo ao nono mês de gestação e GC, ou grupo controle, composto por 15 mulheres não grávidas.

Todas as participantes do estudo assinaram o "Termo de Consentimento Livre e Esclarecido", cujos procedimentos adotados obedecem aos princípios éticos para pesquisa clínica da Declaração de Helsinque e as diretrizes e normas regulamentadoras de pesquisas, envolvendo 
seres humanos, contidas na Resolução 466/2012 do Conselho Nacional de Saúde. Esse estudo esta inserido no projeto "A influencia da cinesioterapia nos parâmetros clinicos e funcionais de gestantes", o qual está de acordo com as orientações do Comitê de Ética em Pesquisa da FCT/UNESP (processo no69/2011).

Os critérios de inclusão para o grupo GG foram: gestantes com consentimento médico e feto único, ausência de diabetes ou outras enfermidades sistêmicas, alterações circulatórias, neuropáticas e/ou vestibulares, doenças musculoesqueléticas anteriores a gestação e intercorrências do ciclo gravídico (RIBAS, GUIRRO, 2007).

As participantes passaram por uma avaliação, realizada pelo mesmo avaliador, para coleta de dados pessoais e antropométricos, e informações quanto à prática de atividade física. Para avaliação da flexibilidade, foram usados o teste de Banco de Wells e o Índice de Schober, que identificam encurtamentos da cadeia posterior e avaliam a mobilidade da região lombossacra.

O Banco de Wells utilizado (Modelo Instant Flex, Sanny ${ }^{\oplus}$, Brasil) possui capacidade de zero a $68 \mathrm{~cm}$ e foi estabilizado contra uma parede. Para o teste, a voluntária permaneceu sentada com as mãos sobrepostas sobre a superfície horizontal do banco, joelhos estendidos e pés descalços, avaliados inicialmente separados e paralelos, respeitando a largura do banco, e realizou-se uma segunda avaliação com os pés juntos e centralizados em relação ao banco. Foi solicitada a flexão anterior da coluna vertebral, com a cabeça entre os braços, sem fletir os joelhos, mantendo-se estática a partir do momento em que conseguiu a posição de máximo alcance do movimento (FARIA, BARROS, 1998). Foram coletadas três medidas com os pés paralelos e três medidas com os pés juntos, sendo utilizada para análise de dados a medida de maior valor de cada uma das formas de se realizar o teste.

O teste de Schober foi realizado em pé, com os joelhos em extensão e pés na posição nominal. Em seguida, traçou-se uma linha unindo as duas espinhas ilíacas póstero-superiores e outra 10 centímetros acima. Foi solicitado que a voluntária realizasse uma flexão anterior do tronco. A partir da flexão anterior, mensurou-se a distância entre as espinhas ilíacas pósterosuperiores e o ponto marcado 10 centímetros acima. Quando a distância aumentou em cinco centímetros ou mais, considerou-se como uma boa mobilidade ou mobilidade normal (MARQUES, 2003). 


\section{RESULTADOS}

Os grupos são compostos por 15 voluntárias cada, sendo que o GG possui gestantes com média de 33,33 \pm 4,27 semanas gestacionais. A caracterização da amostra é apresentada por meio de estatística descritiva expressa em média e desvio padrão, como mostra a Tabela 1. Já a Tabela 2 está relacionada com a prática ou não de atividade física.

Tabela 1. Características (média \pm desvio padrão) das participantes do Grupo Gestante (GG), e Grupo Controle (GC).

\begin{tabular}{lccc}
\hline Variáveis & GG (n=15) & GC (n=15) & \multicolumn{2}{l}{$\begin{array}{l}\text { Valores de } \\
\boldsymbol{p}\end{array}$} \\
\hline Idade (anos) & $26,98 \pm 5,35$ & $26,33 \pm 2,94$ & 0,6282 \\
Altura (m) & $1,65 \pm 0,05$ & $1,63 \pm 0,05$ & 0,7948 \\
IMC Inicial & $22,70 \pm 4,15$ & $24,57 \pm 3,17$ & 0,6016 \\
\hline
\end{tabular}

Tabela 2 - Frequência e porcentagem (\%) de prática de atividade física das participantes do Grupo Gestante (GG) e Grupo Controle (GC).

\begin{tabular}{lcc}
\hline & \multicolumn{2}{c}{ Prática de Atividade Física } \\
Grupos & Sim & Não \\
\hline GG $(\mathbf{n = 1 5})$ & $7(46,67 \%)$ & $8(53,33 \%)$ \\
GC $(\mathbf{n = 1 5})$ & $8(53,33 \%)$ & $7(46,67 \%)$ \\
\hline
\end{tabular}

A tabela 3 demonstra os resultados do Teste de Schober para o GG e GC.

Tabela 3 - Frequência e porcentagem (\%) das participantes do Grupo Gestante (GG) e Grupo Controle (GC) de acordo com a mobilidade lombossacral.

\begin{tabular}{lcc}
\hline & \multicolumn{2}{c}{ Mobilidade Lombossacral } \\
Grupos & Normal $(\geq \mathbf{5} \mathbf{~ c m})$ & Anormal $(<\mathbf{5 c m})$ \\
\hline GG $(\mathbf{n}=\mathbf{1 5})$ & $8(53,33 \%)$ & $7(46,67 \%)$ \\
GC $(\mathbf{n = 1 5 )}$ & $8(53,33 \%)$ & $7(46,67 \%)$ \\
\hline
\end{tabular}

Na tabela 4 seguem os resultados do teste do Banco de Wells realizado com os pés juntos e pés separados. 
Tabela 4. Média e desvio padrão do teste do banco de Wells para o grupo Grupo Gestante (GG) e Grupo Controle (GC).

\begin{tabular}{lccc}
\hline & \multicolumn{3}{c}{ Wells $(\mathbf{c m})$} \\
\cline { 2 - 4 } & Pés separados & Pés juntos & $\begin{array}{c}\mathbf{P} \\
\text { valor }\end{array}$ \\
$\begin{array}{l}\mathbf{G G} \\
\mathbf{( n = 1 5 )}\end{array}$ & $17,33 \pm 10,20 \mathrm{a} * *$ & $14,4 \pm 11,31 \mathrm{a} * *$ & 0,7058 \\
$\mathbf{G C}$ & $32,93 \pm 9,09 \mathrm{~b}$ & $31,87 \pm 9,19 \mathrm{~b}$ & 0,9670 \\
$\mathbf{( n = 1 5 )}$ & & & \\
$\mathbf{P}$ valor & 0,001 & 0,001 & - \\
\hline
\end{tabular}

Letras diferentes na mesma coluna ou linha indicam significância entre os grupos.

$* *$ valores extremamente significativos $(p<0,001)$.

\section{DISCUSSÃO}

O propósito do estudo foi avaliar o grau de flexibilidade de gestantes durante o último trimestre da gestação. A amostra apresentou-se de forma homogênea, o que é demonstrado pela não significância tanto em relação às características de idade quanto aos dados antopométricos de ambos os grupos.

De acordo, com os resultados o GG e o GC não apresentaram diferenças significativas em relação à realização de atividade física, sendo que metade das voluntárias de cada grupo praticam atividades físicas regularmente. Lima e Oliveira (2005) relatam que o sedentarismo é um fator predisponente a doenças durante e após a gestação.

Segundo Moreira et al (2011) a atividade física auxilia no alívio da dor lombar, no restabelecimento da consciência corporal, na adaptação à nova postura e na melhora da resistência e a flexibilidade muscular, reduzindo a sobrecarga sobre os músculos lombares. Além disso, diminui o risco de lesões e melhora a habilidade motora durante o próprio exercício e durante a realização das atividades de vida e de trabalho diários.

Rodrigues et al (2008) ressalta que a atividade física desenvolvida durante a gestação deve ter por características exercícios de intensidade regular e moderada, adaptados e voltados para o período gestacional em que se encontra a mulher.

Neste estudo, o teste de Índice de Schober, que avalia a mobilidade do segmento lombossacral, não apresentou diferenças significativas entre os grupos. Como cita Toscano e Egypto (2001) à flexibilidade diminuída leva a redução da amplitude articular em várias tarefas cotidianas e predispõe a rigidez articular e a limitação do movimento, impossibilitando-o de ser 
realizado sem dor. $\mathrm{O}$ alívio dos sintomas nas lombalgias agudas e crônicas tem sido atribuído à melhora da amplitude do movimento, contudo, essa associação ainda não apresenta base sólida científica.

Já em relação ao teste de flexibilidade da cadeira muscular posterior realizado com o Banco de Wells foi possível observar que houve diferença extremamente significativa entre o GG e o GC, tanto para o teste realizado com os pés separados quanto para o teste realizado com os pés juntos em relação ao grupo controle. Para Sebben et al (2011) a diminuição da flexibilidade ocorre como resultado do crescimento da circunferência abdominal, decorrente da evolução gestacional. Contudo, segundo evidências deste estudo, para avaliação da flexibilidade através do Banco de Wells o teste pode ser executado tanto com os pés juntos quanto separados, a fim de evitar limitações por conta do desenvolvimento da região abdominal.

Para que haja uma boa amplitude de movimento, ou seja, uma boa flexibilidade, é preciso haver mobilidade e elasticidade adequada dos tecidos moles que circundam a articulação. Para que seja alcançada, pode ser utilizada técnica de alongamento como forma de trabalho que visa a manutenção dos níveis de flexibilidade obtidos através da promoção de elasticidade muscular, sendo essa descrita por Badaro et al 2007.

Segundo Dalvi e colaboradores (2010), exercícios que envolvam o alongamento excêntrico contribuem para o alívio da dor lombar e pélvica, promovendo aumento da amplitude de movimento articular e da força muscular, diminuição da tensão dos músculos, melhora da condutividade nervosa e consequentemente diminuição a intensidade da dor. Dessa forma, contribuindo para a promoção de adaptações biomecânicas e manutenção da postura, proporcionando saúde, bem-estar e qualidade de vida.

\section{CONCLUSÃO}

Conclui-se, portanto, que a flexibilidade em gestantes do último trimestre é reduzida quando comparada a mulheres não grávidas. Diante disso, este estudo contribui para o desenvolvimento de atividades preventivas próprias para gestantes, fazendo com que estas participem ativamente de sua gestação, possibilitando a geração de novos estudos relacionados ao tema. 


\section{REFERÊNCIAS}

1. BADARO, A. F. V.; SILVA, A. H.; BECHE, D. Flexibilidade versus Alongamento: esclarecendo as diferenças. Revista Saúde, v. 33, n. 1, p. 32-36, 2007.

2. BIM, C. R.; PEREGO, A. L.; PIRES Jr., H. Fisioterapia aplicada à ginecologia e obstetrícia. Iniciação científica: Cesumar, v. 4, n. 1, p. 57-61, mar./jul., 2002.

3. DALVI, R. A.; TAVARES, E. A.; MARVILA, N. D.; VARGAS, S. C.; NETO, N. C. R. Benefícios da cinesioterapia a partir do segundo trimestre gestacional. Revista Saúde e Pesquisa, v. 3, n. 1, p. 47-51, jan./abr., 2010.

4. FABRIN, E. D.; CRODA, R. S.; OLIVEIRA, M. M. F. Influência das técnicas de fisioterapia nas algias posturais gestacionais. Ensaios e Ciência: Ciências Biológicas, Agrárias e da Saúde, v. 14, n. 2, p. 155-162, 2010.

5. FARIA Jr., .J. C., Barros M. V. G. Flexibilidade e Aptidão Física Relacionada à Saúde. Revista Corporis, v. 3, n. 3, 1998.

6. GAZANEO, M. M.; OLIVEIRA, L. F. Alterações posturais durante a gestação. Rev. Brasileira Atividade Física \& Saúde, v. 3, n. 2, p. 13-21, 1998.

7. LEITÃO, M. B.; LAZZOLI, J. K.; OLIVEIRA, M. A. B.; NÓBREGA, A. C. L.; SILVEIRA, G. G.; CARVALHO, T., et al. Posicionamento oficial da Sociedade Brasileira de Medicina do Esporte: atividade física e saúde na mulher. Rev. Bras. Med. Esporte, v. 6, n. 6, nov/dez, 2000.

8. LIMA, F. R.; OLIVEIRA, N. Gravidez e exercício. Rev. Bras. Reumatol., v. 45, n. 3, p. 188-90, mai./jun., 2005.

9. MANN, L.; KLEINPAUL, J. F.; TEIXEIRA, C. S.; KONOPKA, C. K. Dor lombo-pélvica e exercício físico durante a gestação. Fisioterapia Mov., v.21, n. 2, p. 99-105, abr/jun, 2008.

10. MANN, L; KLEINPAUL, J. F.; MOTA, C. B.; SANTOS, S. G. Alterações biomecânicas durante o período gestacional: uma revisão. Motriz, v. 16, n. 3, p. 730-741, jul/set, 2010.

11. MARQUES, Amélia Pasqual, 1949 - Manual de Goniometria/Amélia Pasqual Marques. 2. ed. Barueri, SP : Manole, 2003.

12. MOREIRA, L. S.; ANDRADE, S. R. S.; SOARES, V.; AVELAR, I. S.; AMARAL, W. N.; VIEIRA, M. F. Alterações Posturais, de equilíbrio e dor lombar no período gestacional. Femina, v. 3, n. 5, maio, 2011.

13. NASCIMENTO, S. L.; SURITA, F. G. C.; PARPINELLI, M. A.; CECATTI, J. G. Exercício físico no ganho de peso e resultados perinatais em gestantes com sobrepeso e obesidade: uma revisão sistemática de ensaios clínicos. Cad. Saúde Pública, v. 27, n. 3, p. 407-416 mar, 2011. http://dx.doi.org/10.1590/S0102-311X2011000300002

14. REIS, G. F. F. Alterações Fisiológicas Maternas da Gravidez. Revista Brasileira de Anestesiologia, v. 43, n. 1, p. 3-9 jan/fev, 1993. 
15. RIBAS, S. I.; GUIRRO, E. C. O. Análise da pressão plantar e do equilíbrio postural em diferentes fases da gestação. Rev. Bras. Fisioterapia, v. 11, n. 5, p. 391-96 set/out, 2007. http://dx.doi.org/10.1590/S1413-35552007000500010

16. RODRIGUES, V. D.; SILVA, A. G.; CÂMARA, C. S.; LAGES, R. J.; ÁVILA, W. R. M. Prática de exercício físico na gestação. Revista Digital, ano 13, n 126, novembro, 2008.

17. SEBBEN, V.; PERUSSOLO, C.; GUEDES, J. M.; TOURINHO, H. F. Tratamento hidroterapêutico na dor lombar em gestantes. Perspectiva, v. 35, n. 129, p. 167-175, março, 2011.

18. TOSCANO, J. O.; EGYPTO, E. P. A influência do sedentarismo na prevalência de lombalgia. Rev. Bras. Med. Esporte, v. 7, n. 4, Jul./Ago., 2001. 